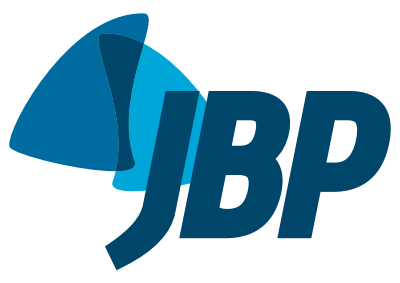

\title{
Does methylene blue attenuate inflammation in nonischemic lungs after lung transplantation?
}

\author{
Marcus da Matta Abreu, ,a, Francine Maria de Almeida, ${ }^{1, b}$, \\ Kelli Borges dos Santos ${ }^{2, c}$, Emílio Augusto Campos Pereira de Assis ${ }^{3, \mathrm{~d}}$, \\ Rafael Kenji Fonseca Hamada ${ }^{4, e}$, Fabio Biscegli Jatene ${ }^{1, f}$, \\ Paulo Manuel Pêgo-Fernandes ${ }^{1, \mathrm{~g}}$, Rogerio Pazetti ${ }^{1, \mathrm{~h}}$
}

1. Departamento de Cardiopneumologia, Disciplina de Cirurgia Torácica, Instituto do Coração, Hospital das Clínicas, Faculdade de Medicina, Universidade de São Paulo, São Paulo (SP) Brasil.

2. Núcleo de Pesquisa em Transplante, Faculdade de Enfermagem, Universidade Federal de Juiz de Fora, Juiz de Fora (MG) Brasil.

3. Departamento de Patologia, Faculdade de Ciências Médicas e da Saúde de Juiz de Fora, Juiz de Fora (MG) Brasil.

4. Faculdade de Medicina, Universidade Federal de Juiz de Fora, Juiz de Fora (MG) Brasil.

a. (D) http://orcid.org/0000-0002-4910-6266

b. (D) http://orcid.org/0000-0001-9755-5034

c. (iD http://orcid.org/0000-0001-8423-9147

d. (D) http://orcid.org/0000-0002-1326-9872

e. (iD http://orcid.org/0000-0002-7540-3141

f. (iD http://orcid.org/0000-0001-6175-5595

g. (iD http://orcid.org/0000-0001-7243-5343

h. (D) http://orcid.org/0000-0001-9823-7783

\begin{abstract}
Objective: To evaluate whether methylene blue (MB) could minimize the effects of ischemia-reperfusion injury in the nonischemic lung on a lung transplantation rodent model. Methods: Forty female Sprague-Dawley rats were divided into 20 donors and 20 recipients. The 20 recipient rats were divided into two groups $(n=10)$ according to the treatment $(0.9 \%$ saline vs. $1 \% \mathrm{MB}$ solutions). All animals underwent unilateral lung transplantation. Recipients received $2 \mathrm{~mL}$ of saline or $\mathrm{MB}$ intraperitoneally prior to transplantation. After $2 \mathrm{~h}$ of reperfusion, the animals were euthanized and histopathological and immunohistochemical analyses were performed in the nonischemic lung. Results: There was a significant decrease in inflammation-neutrophil count and intercellular adhesion molecule-1 (ICAM-1) expression in lung parenchyma were higher in the saline group in comparison with the MB group-and in apoptosis-caspase-3 expression was higher in the saline group and $\mathrm{Bcl}-2$ expression was higher in $\mathrm{MB}$ group. Conclusions: $\mathrm{MB}$ is an effective drug for the protection of nonischemic lungs against inflammation and apoptosis following unilateral lung transplantation in rats.
\end{abstract}

Keywords: Reperfusion injury; Methylene blue; Lung transplantation; Apoptosis; Inflammation.

Submitted: 28 May 2017

Accepted: 7 December 2017

Study carried out in the Departamento de Cardiopneumologia, Disciplina de Cirurgia Torácica, Instituto do Coração, Hospital das Clínicas, Faculdade de Medicina, Universidade de São Paulo,

São Paulo (SP) Brasil.

\section{INTRODUCTION}

The etiology of ischemia-reperfusion injury (IRI) primarily involves the increased formation of reactive oxygen species (ROS). ${ }^{(1,2)}$ A decreased oxygen supply reduces the synthesis and resynthesis of ATP, creating an ionic gradient in the cell membrane due to decreased extracellular active calcium transport. The accumulation of cytoplasmic calcium leads to the activation of a protease that converts xanthine dehydrogenase to xanthine oxidase. ${ }^{(3)}$ Concurrent with these events, there is an accumulation of AMP, which decomposes into substances such as adenosine, inosine, and hypoxanthine. During the reperfusion process, in the presence of oxygen, xanthine oxidase converts hypoxanthine into ROS, such as superoxide, peroxide, and hydroxyl radicals. ${ }^{(3,4)}$ The release of ROS causes cell inflammation and apoptosis of cells as a late phase response of IRI. ${ }^{(5)}$

Methylene blue (MB) prevents ROS production by acting as an alternative xanthine oxidase electron receptor, competing with molecular oxygen for electron transfer. The electrons are transferred to MB from the iron-sulfur center of xanthine oxidase, thus preventing the conversion of molecular oxygen into superoxide radicals.(4)

We previously demonstrated that MB was able to reduce the effects of IRI when we studied transplanted lungs of rats. ${ }^{(6)}$ Some of the free radicals, proteases, and other mediators produced by ischemia and reperfusion after unilateral lung transplantation are washed out and released into the blood stream, and the mediators reach the contralateral lung. There have been few studies on

Correspondence to:

Marcus da Matta Abreu. Rua Deputado Lahyr Tostes, 365, CEP 36037-754, Juiz de Fora, MG, Brasil.

Tel./Fax: 5532 99810-9462. E-mail: marcusdiretoria@hotmail.com

Financial support: This study received financial support from the Fundação de Amparo à Pesquisa do Estado de São Paulo (FAPESP, São Paulo Research

Foundation; Grant no. 2011/50715-0) 
the effect of IRI after single-lung transplantation on the nonischemic lung. ${ }^{(7)}$

The objective of the present study was to evaluate the effects of MB as an inhibitor of IRI on nonischemic right lungs after left lung transplantation in rats.

\section{METHODS}

Forty female Sprague-Dawley rats (300-350 g) were used in the present study (20 donors/20 recipients). Recipient rats were divided into two groups $(n=10)$ according to treatment with saline solution ( $S A L$ ) or $M B$, i.e., SAL group and MB group. The study was approved by our institutional research ethics committee (CAPPesq Protocol no. 3387/09/138) and performed in accordance with the Guide for the Care and Use of Laboratory Animals. ${ }^{(8)}$

\section{Surgical procedure}

\section{Donors}

The animals were anesthetized with $5 \%$ isoflurane (Isothane; Baxter, Jayuya, PR, USA), orotracheally intubated, and mechanically ventilated (model 683; Harvard Apparatus, Holliston, MA, USA) with a volume of $10 \mathrm{~mL} / \mathrm{kg}$ and a respiratory rate of 80 breaths/min. General anesthesia was maintained with $2 \%$ isoflurane (Isovapor model 1224; Takaoka, São Paulo, Brazil). After median laparotomy, $500 \mathrm{U}$ of heparin were injected into the inferior vena cava. After one minute, a median sternotomy was performed, and pulmonary artery was cannulated for anterograde perfusion with $20 \mathrm{~mL}$ of low-potassium dextran (LPD) solution (Perfadex ${ }^{\circledR}$; Vitrolife, Kungsbacka, Sweden) at $4^{\circ} \mathrm{C}$ with constant pressure $\left(20 \mathrm{cmH}_{2} \mathrm{O}\right)$. Prior to perfusion, the inferior vena cava was sectioned to decrease venous return, and the left atrial appendage was amputated to drain the LPD solution. Animals were euthanized by exsanguination in accordance with the American Veterinary Medicine Association. ${ }^{(9)}$

After perfusion, the trachea of the animals was tied at the end of inspiratory flow, and the cardiopulmonary block was excised and placed into a Petri dish with cold LPD for back table procedure. The left hilum was dissected, and cuffs were applied to the artery, vein, and bronchus, as previously described.(10) Grafts were maintained inflated during the ischemia period ( $3 \mathrm{~h}$ ) and were stored in cold LPD until implantation.

\section{Recipients}

Recipient animals were anesthetized, intubated, and ventilated as described above. Immediately prior to graft implantation, animals were intraperitoneally injected with $2 \mathrm{~mL}$ of either $0.9 \% \mathrm{SAL}$ or $1 \% \mathrm{MB}$ solutions. Then, they were placed in right lateral recumbency and subjected to left thoracotomy at the fourth intercostal space. Subsequently, graft implantation was performed using a stereomicroscope (model SZ61; Olympus, Tokyo, Japan) at 8 magnification. ${ }^{(10)}$ In brief, the left hilum was dissected and clamped as proximally as possible. Then, graft implantation was performed by introducing the graft cuffs into a little hole made in the ventral wall of the artery, vein, and bronchus, respectively. After cuff fixation using a 7.0-polypropylene silk suture, the bronchus clamp was slowly opened and air flow was reestablished. In sequence, the vein clamp was removed for retrograde circulation establishment, and, finally, the artery clamp was gently opened, aiming at a soft graft perfusion. The closure of the recipient incision was performed in separate layers using 2.0-monofilament nylon sutures. After surgery completion, animals received analgesia (dipyrone, $400 \mathrm{mg} / \mathrm{kg}$ ) by gavage and were placed under spontaneous ventilation in individual cages with free access to water and food.

Two hours after graft reperfusion, the animals were once more anesthetized, intubated and placed on mechanical ventilation, according to the previously mentioned parameters. The animals were subjected to exploratory laparotomy and euthanasia by incision of the anterior abdominal aorta, with subsequent removal of the cardiopulmonary block. Next, the blocks were stored in $4 \%$ formaldehyde solution over a period of $24 \mathrm{~h}$, and, subsequently, kept in $70 \%$ ethyl alcohol solution until the preparation of the slides for histopathological and immunohistochemical analysis.

\section{Histological analysis}

Both lungs were fixed by tracheal instillation of $4 \%$ formaldehyde solution $\left(20 \mathrm{cmH}_{2} \mathrm{O}\right.$ ) and stored for $24 \mathrm{~h}$ in the same solution for histological analysis. Paraffin-embedded lung samples were cut into $5-\mu \mathrm{m}$ sections and stained with H\&E. Histomorphometry by point-counting technique was used in order to quantify inflammatory cells in lung parenchyma with a Weibel grid containing 100 points and 50 lines. Ten random and mismatched microscopic fields were examined (magnification, $\times 400$ ), totaling 1,000 points per slide and covering an area of $62,500 \mu \mathrm{m}$ per field. ${ }^{(11)}$ The same methodology was used to evaluate the expression of intercellular adhesion molecule-1 (ICAM-1), caspase-3, and $\mathrm{BCl}-2$, which were evaluated through the analysis of slides prepared with a immunohistochemical method described by Almeida et al. ${ }^{(12)}$

\section{Statistical analysis}

Descriptive analysis was performed for quantitative data with normal distribution, and the results were expressed as mean \pm SD. Normality of data distribution and homogeneity of variances were evaluated via the Shapiro-Wilk test and the Levene's test, respectively. The t-test was used for dependent quantitative variables. A type I error of 0.05 (a) was considered for all inferential analyses.

\section{RESULTS}

Regarding inflammatory cells in the lung parenchyma, mean neutrophil counts were higher in the SAL group in comparison with the MB group (5.2 $\pm 2.5 \%$ vs. 
$2.3 \pm 0.8 \% ; p=0.04 ;$ Figure 1$)$, as were ICAM-1 expression ( $4.7 \pm 0.8 \%$ vs. $2.7 \pm 0.7 \%$; $p \leq 0.001$; Figure 2$)$ and caspase-3 expression ( $4.4 \pm 1.2 \%$ vs. $3.0 \pm 1.3 \% ; \mathrm{p} \leq 0.001 ;$ Figure 3). However, Bcl-2 expression in lung parenchyma was higher in the MB group in comparison with the SAL group $(4.9 \pm 1.9 \%$ vs. $2.5 \pm 0.8 \% ; p \leq 0.001$; Figure 4).

Data on the comparisons between the nonischemic lungs (right lungs) and the grafts (left lungs) of the animals in the SAL and in the MB groups are shown in Tables 1 and 2, respectively.

\section{DISCUSSION}

Lung IRI occurs in various cases, such as in cardiopulmonary bypass, lung transplantation, and postenucleation of pulmonary embolism. Recently, much attention has been paid to pulmonary dysfunction resulted from lung IRI. ${ }^{(13)}$

One-lung IRI can lead to similar, but less severe, injury in the contralateral lung. Because injury in the nonischemic lung develops only after reperfusion of the ischemic one, injury is probably humorally mediated. ${ }^{(14)}$ In our study, we were able to confirm this observation,

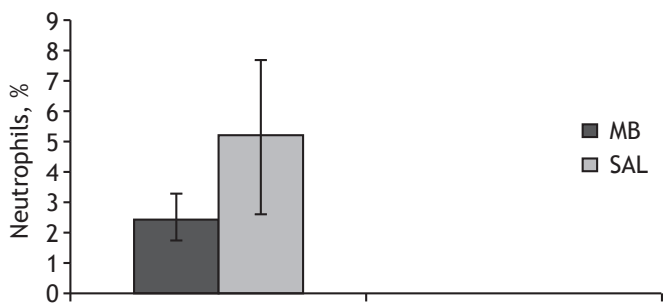

Figure 1. Neutrophil infiltration (\%) in the saline group (SAL) and in the methylene blue (MB) group after 3-h cold ischemic time, transplantation, and 2-h reperfusion in nonischemic lungs after unilateral left lung transplantation. $\mathrm{p} \leq 0,001$.

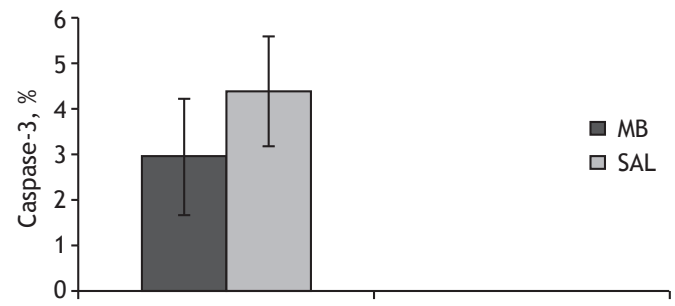

Figure 2. Intercellular adhesion molecule-1 (ICAM-1) activity in the saline group (SAL) and in the methylene blue (MB) group after 3 -h cold ischemic time, transplantation, and 2 -h reperfusion in nonischemic lungs after unilateral left lung transplantation. $p \leq 0,001$. because the native lungs showed a lower expression of inflammatory and apoptotic markers both among the animals submitted to MB instillation and among the animals in the control group.

Contralateral lung injury induced by unilateral lung ischemia and reperfusion is a distinct and complicated phenomenon, which has yet to be fully understood. ${ }^{(15)}$ Some authors have studied the injury of nonischemic lungs after ischemia and reperfusion of the left lung. Zhu et al. ${ }^{(16)}$ used apocynin, an inhibitor of NADPH oxidase, in rats submitted to $60 \mathrm{~min}$ of ischemia by clamping the left pulmonary hilum followed by 30 min of reperfusion. The authors observed that ROS produced by ischemia affected the nonischemic lung. Georgieva et al. ${ }^{(17)}$ concluded that an injured organ affects a remote organ by liberating humoral mediators in an ischemia and reperfusion model similar to that of the study by Zhu et al.(16) To our knowledge, the present study is the first one to assess these effects on nonischemic lungs after pulmonary ischemia and reperfusion induced by unilateral transplantation.

In a previous study, ${ }^{\left({ }^{6}\right)}$ our group evaluated the effects of MB on the lungs of rats submitted to unilateral lung

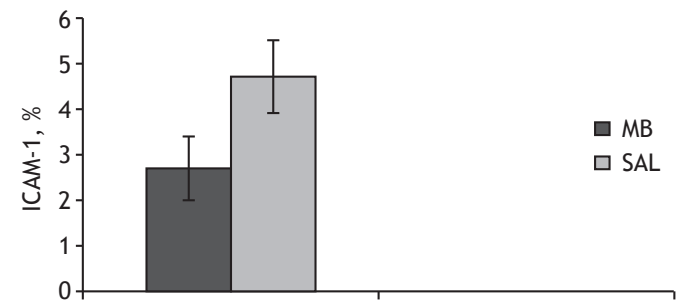

Figure 3. Caspase-3 activity (\%) in the saline group (SAL) and in the methylene blue (MB) group after 3-h cold ischemic time, transplantation, and 2-h reperfusion in nonischemic lungs after unilateral left lung transplantation. $p \leq 0,001$.

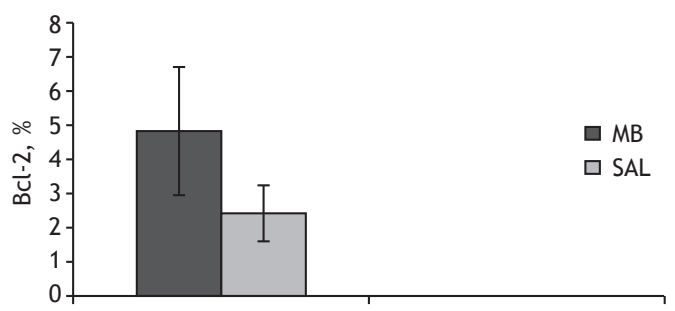

Figure 4. Bcl-2 activity (\%) in the saline group (SAL) and in the methylene blue (MB) group after 3 -h cold ischemic time, transplantation, and 2-h reperfusion in nonischemic lungs after unilateral left lung transplantation. $p \leq 0,001$.

Table 1. Comparison of the degree of inflammation and apoptosis between nonischemic lungs and grafts in the control group.

\begin{tabular}{lccc} 
Variable & Graft (left lung) & Nonischemic lung (right lung) & p \\
Neutrophils, \% & $7.9 \pm 2.0$ & $5.2 \pm 2.5$ & $\leq 0.001$ \\
ICAM-1, \% & $8.1 \pm 2.1$ & $4.7 \pm 0.8$ & $\leq 0.001$ \\
Caspase-3, \% & $6.3 \pm 2.9$ & $4.4 \pm 1.2$ & $\leq 0.001$ \\
Bcl-2, \% & $1.2 \pm 0.9$ & $2.5 \pm 0.8$ & $\leq 0.001$ \\
\hline
\end{tabular}

ICAM-1: intercellular adhesion molecule-1. 
Table 2. Comparison of the degree of inflammation and apoptosis between nonischemic lungs and grafts in the methylene blue group.

\begin{tabular}{lccc}
\multicolumn{1}{c}{ Variable } & Graft (left lung) & Nonischemic lung (right lung) & $\mathbf{p}$ \\
Neutrophils, \% & $4.4 \pm 1.4$ & $2.3 \pm 0.8$ & $\leq 0.001$ \\
ICAM-1, \% & $5.1 \pm 1.1$ & $2.7 \pm 0.7$ & $\leq 0.001$ \\
Caspase-3, \% & $5.7 \pm 1.8$ & $3.0 \pm 1.3$ & $\leq 0.001$ \\
Bcl-2, \% & $2.2 \pm 1.1$ & $4.9 \pm 1.9$ & $\leq 0.001$ \\
\hline
\end{tabular}

ICAM-1: intercellular adhesion molecule-1.

transplantation. As in the present study, MB was able to inhibit neutrophilic infiltration according to the histopathological evaluation. The findings in both studies show that MB, by means of inhibiting the production of ROS, is able to reduce inflammation induced by IRI.

Apoptosis is regulated by a cascade of proteins called caspases, which are activated in ischemia and reperfusion events. Lung ischemia and reperfusion has a direct effect on lung cells, and the increase in caspase- 3 activity reflected a larger number of apoptotic cells. ${ }^{(18)}$

The signaling pathways that lead to apoptosis are maintained by positive and negative regulators. The proteins that promote survival are the antiapoptotic proteins $\mathrm{BCl}-2$ and $\mathrm{BCl}-\mathrm{xL} .{ }^{(19)}$ The release of ROS causes cell apoptosis as a late phase response of IRI. Oxidative stress triggers caspase- 3 activation, leading to cell apoptosis. In addition, the balance of anti- and pro-apoptotic proteins responds dramatically to ROS. ${ }^{(5)}$ Decreased $\mathrm{Bcl}-2$ and increased caspase-3 expressions in the present study are similar to those found by Abogresha et al., (5) who used vitamin C as an antioxidant agent, protecting against the effects of pancreatic injury after renal ischemia. The findings of the present study show the induction of apoptosis as a result of IRI injury after lung transplantation and the ability of MB to inhibit its occurrence.
Endothelial cell adhesion molecules seem to play an important role in IRI by causing adhesion of leukocytes to endothelial cells. ICAM- 1 is one of the adhesion molecules that have been shown to be upregulated in response to cytokines. This upregulation leads to leukocyte-endothelial cell adhesion and to neutrophil infiltration in the affected tissue. Meyer et al.(20) performed 45 min of hepatic ischemia followed by 5 $\mathrm{h}$ of reperfusion and showed a significant upregulation in ICAM-1 in distant organs, such as the heart, kidney, intestine, and pancreas. In the present study, we were able to identify a higher expression of ICAM-1 in the lungs of rats in the SAL group in comparison with those in the MB group, as well as a higher neutrophil count, both of which represent a less intense inflammatory process in the animals treated with MB.

As a limitation of our study, we can highlight that we used the same dose of MB in all cases. MB has a dose-dependent effect, and a study with different doses could have produced different results than those found in the present study. In addition, the monitoring of hemodynamic parameters, markers of tissue perfusion, and ventilation parameters could be useful for a better understanding of the action of MB.

To our knowledge, the present study demonstrates for the first time that MB is an effective drug for the protection of nonischemic lungs against inflammation and apoptosis following unilateral lung transplantation in rats.

\section{REFERENCES}

1. Jatene FB, Pego-Fernandes PM. Challenges in lung transplantation J Bras Pneumol. 2008;34(5):249-50. https://doi.org/10.1590/S180637132008000500001

2. Arcasoy SM, Fisher A, Hashem RR, Scavuzzo M, Ware LB: ISHLT Working Group on Primary Lung Graft Dysfunction. Report of the ISHLT Working Group on Primary Lung Graft Dysfunction part V: predictors and outcomes. J Heart Lung Transplant. 2005,24(10):14838. https://doi.org/10.1016/j.healun.2004.11.314

3. den Hengst WA, Gielis JF, Lin JY, Van Schil PE, de Windt LJ, Moens AL. Lung ischemia-reperfusion injury: a molecular and clinical view on a complex pathophysiological process. Am J Physiol Heart Circ Physiol. 2010;299(5):H1283-99. https://doi.org/10.1152/ ajpheart.00251.2010

4. Greca FH, Gonçalves NM, Souza Filho ZA, Noronha Ld, Silva RF, Rubin MR. The protective effect of methylene blue in lungs, small bowel and kidney after intestinal ischemia and reperfusion. Acta Cir Bras. 2008;23(2):149-56. https://doi.org/10.1590/S010286502008000200007

5. Abogresha NM, Greish SM, Abdelaziz EZ, Khalil WF. Remote effect of kidney ischemia-reperfusion injury on pancreas: role of oxidative stress and mitochondrial apoptosis. Arch Med Sci. 2016;12(2):25262. https://doi.org/10.5114/aoms.2015.48130

6. Abreu Mda M, Pazetti R, Almeida FM, Correia AT, Parra ER, Silva $L P$, et al. Methylene blue attenuates ischemia-reperfusion injury in lung transplantation. J Surg Res. 2014;192(2):635-41. https://doi. org/10.1016/j.jss.2014.07.043

7. Watanabe A, Kawaharada N, Kusajima K, Komatsu S, Takahashi $\mathrm{H}$. Contralateral lung injury associated with single-lung ischemiareperfusion injury. Ann Thorac Surg. 1996;62(6):1644-9. https://doi. org/10.1016/S0003-4975(96)00810-7

8. National Research Council; Division on Earth and Life Studies; Institute for Laboratory Animal Research; Commission on Life Sciences. Guide for the Care and Use of Laboratory Animals [monograph on the Internet]. Washington, DC: The National Academies Press; 1996 [cited 2010 Jan 22]. Available from: https:/ www.nap.edu/catalog/5140/guide-for-the-care-and-use-of-laboratoryanimals

9. Beaver BV, Reed W, Leary S, McKiernan B, Bain F, Schultz R, et al. Report of the AVMA panel on euthanasia. J Am Vet Med Assoc 2001;218:669-696. https://doi.org/10.2460/javma.2001.218.669

10. Mizuta T, Kawaguchi A, Nakahara K, Kawashima Y. Simplified rat lung transplantation using a cuff technique. J Thorac Cardiovasc Surg. 1989;97(4):578-81.

11. Prado CM, Leick-Maldonado EA, Yano L, Leme AS, Capelozzi VL, Martins MA, et al. Effects of nitric oxide synthases in chronic allergic airway inflammation and remodeling. Am J Respir Cell Mol Biol. 2006;35(4):457-65. https://doi.org/10.1165/rcmb.2005-03910C

12. Almeida FM, Oliveira-Junior MC, Souza RA, Petroni RC, Soto SF Soriano FG, et al. Creatine supplementation attenuates pulmonary and systemic effects of lung ischemia and reperfusion injury. $J$ Heart Lung Transplant. 2016;35(2):242-50. https://doi.org/10.1016/j. healun.2015.06.012 
13. Zhao Q, Wu J, Hua Q, Lin Z, Ye L, Zhang W, et al. Resolvin D1 mitigates energy metabolism disorder after ischemia-reperfusion of the rat lung. J Transl Med. 2016;14:81. https://doi.org/10.1186/ s12967-016-0835-7

14. Palazzo R, Hamvas A, Shuman T, Kaiser L, Cooper J, Schuster DP. Injury in nonischemic lung after unilateral pulmonary ischemia with reperfusion. J Appl Physiol (1985). 1992;72(2):612-20. https://doi. org/10.1152/jappl.1992.72.2.612

15. Yeh DY, Tung SP, Fu YH, Yang YC, Wang JJ. Intravenous superoxide dismutase administration reduces contralateral lung injury induced by unilateral lung ischemia and reperfusion in rats through suppression of activity and protein expression of matrix metalloproteases. Transp Proc. 2015;47(4):1083-6. https://doi.org/10.1016/j. transproceed.2014.10.060

16. Zhu C, Bilali A, Georgieva GS, Kurata S, Mitaka C, Imai T. Salvage of nonischemic control lung from injury by unilateral ischemic lung with apocynin, a nicotinamide adenine dinucleotide phosphate
(NADPH) oxidase inhibitor, in isolated perfused rat lung. Transl Res. 2008;152(6):273-82. https://doi.org/10.1016/..trsl.2008.10.008

17. Georgieva GS, Kurata S, Ikeda S, Eishi Y, Mitaka C, Imai T. Nonischemic lung injury by mediators from unilateral ischemic reperfused lung: ameliorating effect of tumor necrosis factor-alphaconverting enzyme inhibitor. Shock. 2007;27(1):84-90. https://doi. org/10.1097/01.shk.0000235131.89986.45

18. Forgiarini LA, Grün G, Kretzman NA, Kretzmann NA, de Mu-oz GA, de Almeida A, Forgiarini LF, et al. When is injury potentially reversible in a lung ischemia-reperfusion model? J Surg Res. 2013;179(1):168-74. https://doi.org/10.1016/j.jss.2012.08.026

19. Ferrari RS, Andrade CF. Oxidative Stress and Lung IschemiaReperfusion Injury. Oxid Med Cell Longev. 2015;2015:590987.

20. Meyer K, Brown MF, Zibari G, Panes J, McMillan RW, McDonald JC, et al. ICAM-1 upregulation in distant tissues after hepatic ischemia/ reperfusion: a clue to the mechanism of multiple organ failure. Pediatr Surg. 199:33(2):350-3. 